\title{
ORIGINAL
}

\section{ANÁLISIS DE LA COMUNICACIÓN EN REDES SOCIALES DE LA CAMPAÑA DE LA VACUNA DE GRIPE EN ESPAÑA}

Ma Isabel Cano Garcinuño (1) y Sergio Arce García (2)

(1) Servicio de Salud del Principado de Asturias (SESPA). Oviedo. España.

(2) Escuela Superior de Ingeniería y Tecnología (ESIT). Universidad Internacional de La Rioja (UNIR). Logroño. España.

Los autores declaran que no existe conflicto de intereses.

\section{RESUMEN}

Fundamentos: Tras la aparición y difusión de los grupos anti-vacunas, se hace necesario realizar campañas de comunicación orientadas a la ciudadanía sobre los beneficios de la vacunación, y las redes sociales son una buena vía para alcanzar a una gran cantidad de población. Este artículo tuvo como objetivo analizar la comunicación en la red social Twitter durante la campaña de la vacuna de la gripe en el año 2018.

Métodos: Se emplearon métodos de big data para recoger la totalidad de tuits sobre la vacuna de la gripe del 23 de octubre al 15 de diciembre de 2018. Se determinaron por análisis de cluster y los cálculos del eigenvector y pagerank quiénes fueron los usuarios o influencers más importantes durante la campaña.

Resultados: Se recogieron un total de 9.147 tuits, de los cuales el $71,94 \%$ fueron retuits (RT) o reenvíos de lo que escriben otros usuarios. Diez grupos generaron el 69,92\% del tráfico de los mensajes sobre vacunas. La emoción principal vertida en los mensajes sobre vacunas fue el miedo a las consecuencias si la gente no se vacunara.

Conclusiones: Se determina que la información sobre la campaña es favorable a la vacunación pero está dirigida principalmente por (supuestamente) médicos, enfermeras o enfermos anónimos que tuitean y son muy seguidos por multitud de usuarios. Las campañas oficiales e institucionales, siendo algunas de ellas redifundidas de forma posiblemente organizada, quedan muy relegadas del seguimiento de la sociedad en las redes.

Palabras clave: Vacunación, Gripe, Comunicación, Red social, Influencer, Twitter.

\section{ABSTRACT \\ Analysis of communication in social networks of the influenza vaccine campaign in Spain}

Background: After arising of anti-vaccine groups and their dissemination, it is necessary to carry out communication campaigns on the benefits of vaccination aimed at citizens, and social networks are a good way to reach a large population. The objective of this article is to determine the communication on Twitter social network during the influenza vaccine campaign in 2018 in Spain.

Methods: Big data methods were used to collect all tweets about the influenza vaccine during October 23 to December 15. They were determined by cluster analysis, eigenvector and pagerank calculations to determinate who were the most important influencers during the campaign.

Results: A total of 9,147 tweets were collected, of which $71.94 \%$ were retweets (RT). Ten groups generated $69.92 \%$ of the message traffic on vaccines. The main emotion expressed in the messages about vaccines is the fear of consequences if people do not get vaccinated.

Conclusions: It was determined that the information on the campaign is favorable to vaccination but is mainly directed by (supposedly) doctors, nurses or anonymous patients who tweet and are followed by many users. The official and institutional campaigns, some of which are re-disseminated in a possibly organized way, are very neglected in the monitoring of society in the networks.

Key words: Vaccination, Flu, Communication, Social network, Influencer, Twitter.

\footnotetext{
Cita sugerida: Cano Garcinuño MI, Arce García S. Análisis de la comunicación en redes sociales de la campaña de la vacuna de gripe en España. Rev Esp Salud Pública. 2020; $94: 2$ de marzo e202003008.
} 


\section{INTRODUCCIÓN}

La gripe es una enfermedad vírica estacional provocada por el virus de la influenza que apareció de forma muy virulenta varias veces a lo largo de la historia. En 1918 tuvo lugar una pandemia con consecuencias devastadoras que recibió el nombre de "gripe española", haciendo referencia al lugar donde se creía que se había originado, que causó la muerte de más de $50 \mathrm{mi}$ llones de personas en todo el mundo. En 2009 se originó en México una nueva gripe provocada por una nueva cepa del virus gripal H1N1, considerada como una pandemia al tratarse de una enfermedad con propagación a nivel mundial. Es muy difícil prever dónde y cuándo se originará la próxima pandemia, así como cuáles serán sus consecuencias ${ }^{(1,2)}$.

De los tres tipos de virus de la gripe conocidos (A, B y C) actualmente se introducen en las vacunas cepas de virus de los dos primeros, puesto que los virus del tipo $\mathrm{C}$ son menos frecuentes y suelen causar infecciones leves, careciendo de importancia desde el punto de vista de la salud pública. Debido a los cambios y mutaciones que sufren estos virus, la persona que haya pasado la enfermedad no queda inmunizada frente a ella, por lo que la vacunación debe repetirse de forma anual ${ }^{(1,3)}$.

La vacunación es uno de los principales logros de la salud pública en el siglo XX, con una gran evidencia científica que demuestra sus beneficios. Es una de las medidas más eficaces para la prevención de la gripe, enfermedad que causa cada año entre 250.000 y 500.000 muertes a nivel mundial. A pesar de la efectividad de la vacunación contra la gripe, la cobertura se mantiene en niveles bajos, siendo inferior al $30 \%$ en la población general de la Unión Europea y en torno al $18 \%$ de los mayores de 15 años en España ${ }^{(4,5,6)}$.

Según datos del Ministerio de Sanidad, en la última campaña de vacunación de la gripe fueron vacunadas un total de 5.373.702 personas, considerando a la población mayor de 60 años, las embarazadas y el personal sanitario, todos ellos incluidos dentro de las recomendaciones de vacunación frente a la gripe del Consejo Interterritorial del Sistema Nacional de Salud (CISNS). Según estos datos, la cobertura fue superior entre los mayores de 65 años que en el resto de los grupos, llegando al 54,2\% del total de la población mayor de 65 años ${ }^{(6)}$.

A pesar de la evidencia científica sobre los beneficios de la vacunación frente a las enfermedades, en los últimos años se está produciendo la reaparición de los movimientos anti-vacunas, aumentando la desconfianza de la población ante esta medida de protección. Por este motivo, es necesario la aplicación urgente de medidas efectivas para aumentar la confianza que tiene la población general en las vacunas. Es importante estudiar los motivos por los que sienten esa desconfianza hacia ellas y seguir una estrategia de concienciación. De esta forma, se conseguirá incrementar las tasas de vacunación existentes en la actualidad ${ }^{(4,5,7)}$.

Con el objetivo de ayudar a los Estados Miembros de la Unión Europea a diseñar sus estrategias de comunicación sobre la campaña de vacunación de la gripe, los Centros Europeos para el Control de Enfermedades (ECDC) editan una guía de apoyo con ejemplos de actividades de comunicación utilizadas en otros países y materiales producidos por los ECDC, UNICEF y la Organización mundial de la salud (OMS). Es necesario que la comunicación se base en mensajes con evidencia científica ${ }^{(5,8)}$. En casos concretos como las mujeres embarazadas, en las que está indicada la vacuna contra la gripe, se observa una baja tasa de vacunación debido principalmente a la falta de información por parte de los profesionales que realizaban la vigilancia y control del embarazo ${ }^{(9)}$.

El uso de Internet en la búsqueda de información relacionada con temas de salud por parte de 
los usuarios supuso un gran cambio en la forma de obtención de dicha información. Distintos medios como blogs, Facebook, Twitter y distintos canales de Youtube son utilizados por muchos usuarios tanto para conseguir información como para difundir sus ideas ${ }^{(10,11)}$.

El uso de Internet y de las redes sociales está aumentando mucho en los últimos años. Según el informe We Are Social Digital 2019, en España existen 42,96 millones de usuarios de Internet, de los que 28 millones son usuarios activos de redes sociales, lo que representa el $60 \%$ de la población total. En dicho informe se observa un crecimiento anual del uso de redes sociales del $3,7 \%$, dedicándose de media una hora y treinta y nueve minutos al día ${ }^{(12)}$. Twitter se posiciona como la tercera red social en España, donde un $46,4 \%$ de usuarios manifiestan emplearla ${ }^{(13)}$. Al igual que en otras redes sociales, en ella se promueven nuevas formas de participación ciudadana, donde la posibilidad de interacción y bidireccionalidad suponen un cambio a la hora de emitir y difundir los mensajes ${ }^{(14)}$. De esta forma, Twitter se convirtió en un lugar de encuentro social donde se intercambian ideas, información y conocimientos $^{(15)}$ pero, como contrapartida, es un lugar de acceso a fuentes no legítimas que pueden introducir dentro del discurso mensajes paralelos en temas de interés social ${ }^{(16)}$.

El objetivo principal de este estudio fue analizar la campaña de la vacunación de la gripe de 2018 en la red social Twitter en España, a través de los siguientes objetivos secundarios: conocer a los usuarios que generaban contenido, descubrir si las administraciones públicas y/o las asociaciones de profesionales lideraban la información a través de las redes y determinar qué tipo de mensaje es el que se enviaba. El Ministerio de Sanidad y la mayoría de las comunidades autónomas establecen campañas de difusión sobre vacunación en redes sociales (https://www.mscbs.gob.es/campannas/ campanas19/home.htm), por lo que quiso determinarse su capacidad de influencia.

\section{MATERIAL Y MÉTODOS}

La captura de mensajes procedentes de la red social Twitter se realizó mediante programación a través del software estadístico $\mathrm{R}$ en su entorno visual Rstudio (en su versión 1.1.456). A través de la librería Rtweet (versión 0.6.7) ${ }^{(17)}$, y la conexión con un interfaz de programación de aplicaciones (API) para programadores de Twitter, se realizó la búsqueda de la totalidad de tuits durante el periodo del 23 de octubre de 2018 a las 10:23 a.m. hasta el 15 de diciembre de 2018 a las 10:16 a.m., abarcando las fechas de periodo de la campaña de vacunación de la gripe en las distintas comunidades autónomas españolas.

Una vez recogidos todos los tuits, se les aplicaron diversos análisis propios del machine learning:

Análisis de redes: a través del software informático Gephi (versión 0.9.2) se introdujeron los datos para realizar un análisis gráfico de la red existente entre usuarios. De esta manera, se representaron a los usuarios o nodos de la red y sus conexiones, mostrando el tamaño del nodo o usuario y su alias en la red en función de su importancia en la misma y el grosor de líneas por su cantidad de conexiones, posicionando y separando los nodos en el gráfico a una distancia en función de la similitud o no entre los usuarios con la aplicación secuencial de los algoritmos OpenOrd y Yifan $\mathrm{Hu}^{(18)}$. Se determinaron igualmente las conexiones entre nodos mediante algoritmos estadísticos de eigenvector o vector central, que miden la importancia de un nodo con respecto a su conexión con el resto de la red, y también el pagerank para clasificarlos de acuerdo a la frecuencia con la que un usuario cualquiera y sin conocimientos previos, siguiendo enlaces de la red, llegase al mensaje en cuestión con una probabilidad del $85 \%{ }^{(19)}$.

Análisis de emociones: fue la forma de medir los discursos de la opinión pública, la determinación 
y el cálculo emocional, que cada vez tiene más importancia ${ }^{(20)}$, mediante la identificación de sentimientos básicos en los discursos: alegría, tristeza, enfado, miedo, disgusto, sorpresa, confianza $\mathrm{y}$ anticipación $\mathrm{o}$ pensamiento racional $^{(21,22)}$. De esta manera, se contrastaron y asignaron a cada tuit las emociones ligadas, utilizando para este análisis el léxico desarrollado por el National Research Council of Canada (versión 0.92) en su versión en castellano ${ }^{(23)}$.

\section{RESULTADOS}

La recogida de tuits durante el periodo de análisis ofreció un resultado de 9.147 tuits en total. La distribución del total no fue uniforme a lo largo del periodo analizado, centrándose en los primeros días y reduciéndose mucho, hasta casi ser inexistente, en las últimas fechas, tal como puede observarse en la figura 1.
Los tuits que se pudieron determinar como de autoría propia fueron $2.572(28,12 \%)$, y resultaron retuits (RT) o reenvíos de lo que escribían otros usuarios un total de 6.576 mensajes $(71,94 \%)$, produciéndose de forma más intensa en las primeras semanas, sobre todo la primera de noviembre, para descender a lo largo de los días hasta casi ser inexistentes. Por lo tanto, cabe destacar que, aunque el mensaje creado por quien escribía no era despreciable, lo que abundó principalmente fue la adhesión a lo que decían otros. El grado medio de retuits por usuario fue de 1.026, por lo que en un principio no cabría ver una insistencia en difusión por cada cuenta. La determinación de los retuits por asociación de cluster determinó que existía una modularidad de 0,776 , un valor de buena asociación de las cuentas a diferentes grupos en redes sociales, y la presencia de 327 comunidades de opinión. De entre todas ellas, las diez
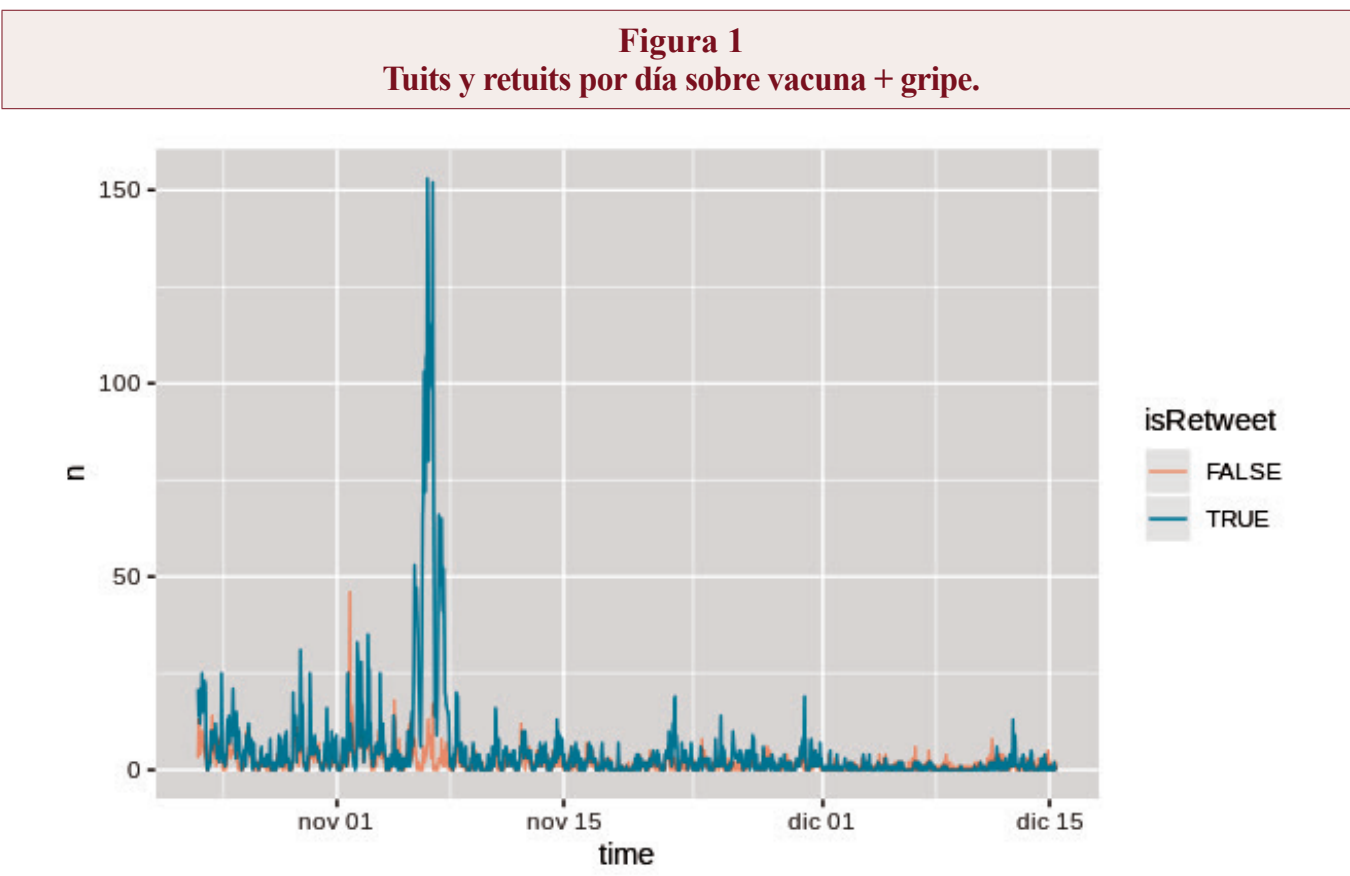

Fuente: Elaboración propia a partir de datos extraídos de Twitter. 
primeras con más influencia entre los usuarios ya supusieron el $69,92 \%$ del total de retuits, donde solo al primer grupo ya correspondió más del 43\% del tráfico de mensajes.

Las comunidades de usuarios detectadas presentaban unos influencers claros que determinaban la opinión, que era reenviada por multitud de otras cuentas y que se muestran en la tabla 1. La comunidad principal que generó opinión sobre la vacuna de la gripe giraba alrededor de los tuits de la cuenta (a)dellnframundo, correspondiente a un usuario que se definía como "enfermera en un Centro de Atención a Drogodependientes" y que fue creada en agosto de 2018, presentando un total de 679 seguidores. Los comentarios de dicha cuenta presentaron alrededor del $43 \%$ del tráfico de tuits sobre la vacuna de la gripe durante su campaña.

En segundo lugar se encontró la cuenta @,AEV_Vacunas, cuenta oficial de la Asociación
Española de Vacunología y responsables de la web vacunas.org, generando un 9,2\% del tráfico de tuits. En tercer lugar apareció la cuenta@JuanGrvas, que se definía como "médico general y Profesor visitante Salud Internacional"; en cuarto, @microBIOblog, un blog sobre noticias científicas; en quinto, @kekoarteixo, un paciente de diálisis y pensionista jubilado; en sexto, @CNNEE, el medio de comunicación CNN en español; en séptimo, @SanofiPasteurLA, una empresa farmacéutica; en octavo, @abianmm, un usuario que hablaba sobre infecciones pediátricas; en noveno, @ HUReinaSofia, el hospital Reina Sofía de Córdoba (Andalucía); eldécimo,@materiaciencia,perteneciente al área de ciencia del diario El País.

Se pudo comprobar así que, de las diez cuentas con más influencia sobre la campaña de la gripe, solo había dos cuentas oficiales de instituciones, apareciendo además una empresa farmacéutica, dos medios de comunicación y un blog de ciencia.

\begin{tabular}{|c|c|c|c|c|}
\hline \multicolumn{5}{|c|}{ Comunidades más importantes de opinión. } \\
\hline Grupo & $\begin{array}{c}\text { Influencer } \\
\text { principal }\end{array}$ & $\begin{array}{c}\text { Porcentaje de } \\
\text { tráfico generado }\end{array}$ & $\begin{array}{c}\text { Eigenvector - } \\
\text { Centralidad }\end{array}$ & Pagerank \\
\hline 1 - rosa & @del1nframundo & $43,03 \%$ & 1,000 & 0,208 \\
\hline 2 - verde & @AEV_Vacunas & $9,20 \%$ & 0,033 & 0,004 \\
\hline 3 - azul & @JuanGrvas & $4,43 \%$ & 0,063 & 0,012 \\
\hline 4 - marrón & @microBIOblog & $3,14 \%$ & 0,019 & 0,004 \\
\hline 5 - naranja & @kekoarteixo & $1,97 \%$ & 0,036 & 0,007 \\
\hline 6 - rojo & @CNNEE & $1,83 \%$ & 0,037 & 0,009 \\
\hline 7 - verde oscuro & @SanofiPasteurLA & $1,68 \%$ & 0,029 & 0,006 \\
\hline 8 - rosa claro & @abianmm & $1,66 \%$ & 0,018 & 0,003 \\
\hline 9 - amarillo & @HUReinaSofia & $1,58 \%$ & 0,016 & 0,003 \\
\hline 10 - azul claro & @materiaciencia & $1,4 \%$ & 0,022 & 0,005 \\
\hline
\end{tabular}


El resto, con porcentajes muy importantes y destacados, eran de personal presuntamente sanitario y pacientes no identificados.

La representación gráfica de las conexiones entre los tuits de los usuarios, representando su grado de importancia por el tamaño de su nodo o círculo y el tamaño del texto del nombre de la cuenta (figura 2), muestra que los usuarios seguían a su cuenta influencer y no presentaban relación con otras cuentas ni conversaciones. De esta manera, cabe destacar que los usuarios seguían a una fuente y no a otras, y solo unos pocos se conectaban entre el primer y segundo grupo de cluster.

El estudio estadístico de la centralidad de la opinión, o eigenvector, giró alrededor de la cuenta de@dellnframundo, quedando el discurso más cercano a la red de @JuanGrvas y bastante alejado del resto de grupos. Justamente la determinación del pagerank, o probabilidad al 85\% de llegar a un discurso por parte de un usuario cualquiera que entrara en Twitter sin conocer lo existente, llegaría primero a los discursos de dichas dos cuentas $(20,8 \%$ y $1,2 \%$ respectivamente).

Un análisis del grado de salida de los usuarios permitió determinar si existía un grupo de cuentas que se dedicaran a retuitear en numerosas ocasiones el discurso de un influencer, mostrando una posible organización en su difusión. Según se muestra en la figura 3, el grupo 2 alrededor de la cuenta @AEV_Vacunas presentó una estructura de diversos usuarios que retuiteaban en diversas $\mathrm{y}$, a veces, numerosas

\section{Figura 2}

Grafo de representación de tuits alrededor de sus influencers.

\section{@del1nframundo}

Fuente: Elaboración propia. 


\section{Figura 3}

\section{Grafo de representación de tuits por grado de salida y ordenados por alturas mediante algoritmo 3D.}

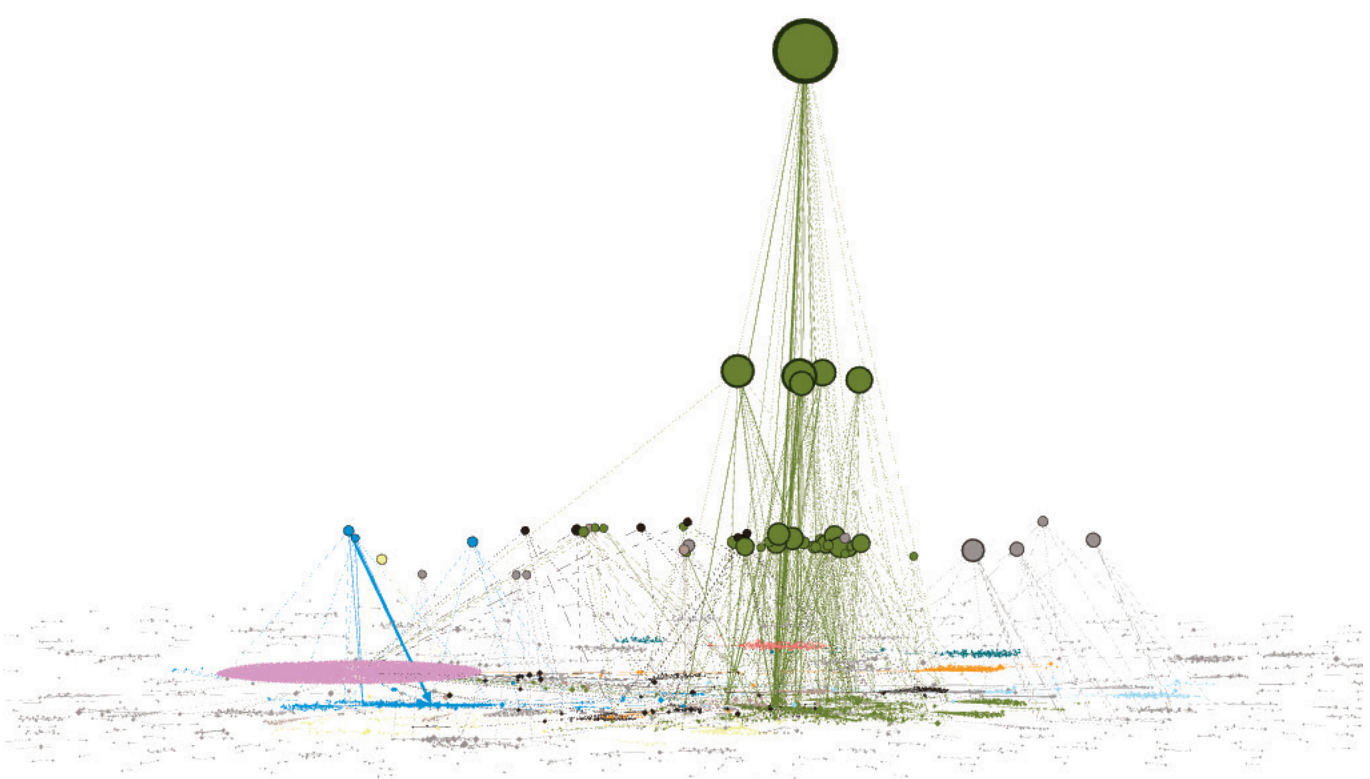

Fuente: Elaboración propia.

ocasiones. Esto indicaría una organización en la difusión por parte de dicha comunidad, que podía deberse a que se trataba de una institución con muchos usuarios que trabajaban allí o que participaban en la misma, dando a conocer sus actividades y ayudando en la redifusión de mensajes, sin apreciar más estructuras en los restantes grupos.

Un análisis de sentimientos de los tuits vertidos, mostrados en la figura 4, ofrece una similitud entre aquellos positivos y los negativos, aunque se observaron algunos polarizados muy negativos, aunque también muy positivos. En cuanto a las emociones básicas encontradas, se detectaron diferencias apreciables. En primer lugar, lo que más apareció fue el miedo (en un $50 \%$ ), así como la anticipación o pensamiento racional (25\%), quedando el resto de emociones en posiciones muy bajas y casi despreciables, por debajo del 10\%. La dispersión de diferentes emociones en los tuits se produjo principalmente con las dos más destacadas, principalmente en la anticipación. El resto de emociones fueron bastante homogéneas y constantes entre los diferentes tuits.

$\mathrm{Si}$ se determinan las emociones de los principales grupos por separado, pueden apreciarse diferencias significativas: mientras que los mensajes alrededor de la cuenta institucional de @,AEV_vacunas presentaron un $55 \%$ de mensajes positivos y un $51 \%$ de discurso de miedo (si uno no se vacunaba), estando el resto de emociones por debajo del $11 \%$, en el grupo de (a)dellnframundo presentaron un mensaje muy neutro ( $2 \%$ de mensajes en formato positivo y $2 \%$ en negativo), con un $99 \%$ de anticipación y un 2\% de miedo. En el de @JuanGrvas apareció un $49 \%$ positivo, $46 \%$ de miedo, $15 \%$ enfado y 


\section{Figura 4}

Análisis de sentimientos y emociones.
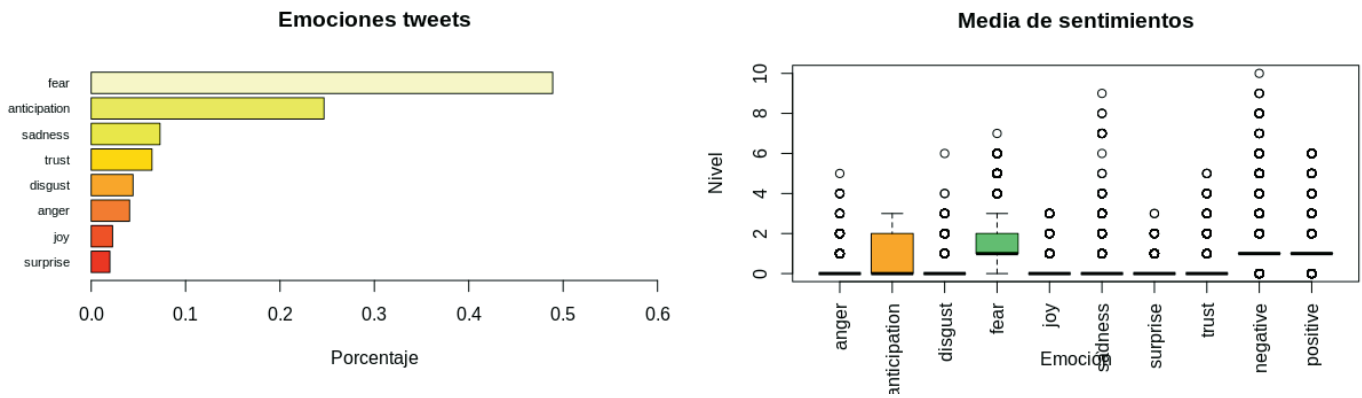

Fuente: Elaboración propia.

10\% tristeza, y en el de @ SanofiPasteurLA un $76 \%$ negativo (como forma de expresión, no por posicionarse en contra de las vacunas) y un $75 \%$ de miedo.

Con respecto al tipo de usuarios que participaban, no se detectaron cuentas que fueran o difundieran mensajes de movimientos anti-vacunas.

\section{DISCUSIÓN}

El análisis de lo vertido en la red social Twitter a lo largo del periodo de la campaña de la gripe en 2018 en las distintas comunidades autónomas españolas muestra que las campañas institucionales o las llamadas de los organismos públicos a la vacunación de la gripe son casi inexistentes o no tienen repercusión suficiente. Aunque desde el Ministerio de Sanidad y la mayoría de las comunidades autónomas se realizan campañas que se difunden por redes sociales, el discurso entre los usuarios supone, principalmente, el seguimiento a través de retuits de determinados creadores de opinión o influencers, que en su mayor parte determinan la opinión y la necesidad de la vacunación. Por tanto, las campañas institucionales apenas tienen repercusión frente a fuentes no oficiales, y así la comunicación se escapa de fuentes fiables hacia otras no controladas, de las que se desconoce casi todo pero que pueden influir en gran medida sobre la opinión pública. Desde determinadas cuentas de enfermeras, médicos o pacientes, sin posibilidad de verificar su identidad verdadera, se establecen los principales mensajes, llegando una de dichas cuentas a generar más del $43 \%$ del tráfico de tuits y siendo el eje principal del establecimiento de opinión en la red social. A gran distancia, entre los diez principales creadores de opinión se puede encontrar a dos instituciones, la Asociación Española de Vacunología y el Hospital Reina Sofía de Córdoba, teniendo la primera un grupo de usuarios que se dedican a redifundir el mensaje original de su fuente principal en numerosas ocasiones y que podrían estar relacionados de forma organizada. Igualmente, se pueden encontrar creadores de mensajes a partir de blogs o de medios de comunicación, pero desde áreas de ciencia no expresamente sanitaria.

Se detecta un porcentaje similar de tuits expresados de forma negativa y positiva, aunque existen unos pocos mensajes o muy negativos o muy positivos. Las emociones se centran principalmente en el miedo y al pensamiento racional, por lo que se está lanzando la vacuna no como algo bueno, sino como una exposición a lo que puede suceder si no se pone de forma argumentada y razonada. 
El principal mensaje de expresión de miedo procede del grupo alrededor de una cuenta de una farmacéutica $(76 \%)$ y desde la principal cuenta institucional de la Asociación Española de Vacunas (51\%), mientras que los influencers aportan mensajes mucho más neutros en sus sentimientos y emociones, y más fuertes en el pensamiento racional. No se detectan mensajes procedentes de cuentas de grupos anti-vacunas.

Se concluye por tanto que el mensaje que se transmite sobre la vacunación de la gripe es que se trata de una necesidad y algo beneficioso, pero el mensaje es manejado principalmente por cuentas propias de personas supuestamente vinculadas al ámbito sanitario, a título personal, así como áreas del ámbito de los blogs y los medios de comunicación de ciencias. Desde el área sanitaria solo aparecen de manera destacable dos instituciones, una de ellas difundiendo posiblemente de forma organizada, a través de sus empleados y colaboradores. Las instituciones públicas o bien no utilizan las redes sociales para llegar a la población o bien su difusión es muy escasa, quedando el mensaje principal de la campaña de la vacunación en manos de personas altruistas.

Las limitaciones de este estudio vienen determinadas por dos vías. La principal proviene de la utilización de la red social Twitter por parte de población joven y, en menor medida, de mediana edad, pero con escaso uso por parte de personas de edad avanzada. Igualmente, la caracterización de emociones mediante procesos de machine learning con el uso de léxicos preasignados no tiene, por el momento, buenos modelos en español, sino que son procedentes de traducciones adaptadas del inglés, por lo que el uso de lenguaje irónico o con localismos puede no estar recogido convenientemente.

\section{BIBLIOGRAFÍA}

1. OMS. Gripe. Disponible en https://www.who.int/topics/ influenza/es.
2. Woodland DL. Building a Better Flu Vaccine. Viral Immunol 2018;31(4):277.

3. Comité asesor de vacunas de la AEP. Vacuna de la gripe. Disponible en https://vacunasaep.org/familias/vacunas-unaa-una/vacuna-gripe.

4. Eskola J, Duclos P, Schuster M, MacDonald NE. How to deal with vaccine hesitancy? Vaccine 2015;33:4215-4217.

5. Ohlrogge AW, Suggs LS. Flu vaccination communication in Europe: What does the government communicate and how? Vaccine 2018;36(44):6512-6519.

6. Ministerio de Sanidad, Consumo y Bienestar Social. Disponible en https://www.mscbs.gob.es/profesionales/saludPublica/prevPromocion/vacunaciones/home.htm .

7. Thomson A, Vallee-Tourangeu G, Suggs LS. Strategies to increase vaccine acceptance and uptake: from behavioral insights to context-specific, culturally-appropriate, evidence-based communications and interventions. Vaccine 2018;36(44):6457-6458.

8. ECDC. Seasonal influenza communication toolkit guidelines. 2016. Disponible en https://ecdc.europa.eu/en/seasonalinfluenza/prevention-and-control/communication-toolkit.

9. Tuells J, Rodríguez-Blanco N, Duro Torrijos JL, Vila-Candel $\mathrm{R}$, Nolasco Bonmati A. Vaccination of pregnant women in the Valencian Community during the 2014-15 influenza season: a multicentre study. Rev Esp Quimioter 2018;31(4):344-352.

10. Cuesta U, Gaspar S. La "reputación online" de la información de vacunas en internet. Historia y comunicación social 2014;19:15-29.

11. Cuesta U, Gaspar S. Comunicación 2.0 y salud pública: redes sociales, "influencers" y vacunas. En: Innovación universitaria: digitalización 2.0 y excelencia en contenidos. Madrid, McGraw-Hill; 2016.p.161-175.

12 Digital 2019 España. Madrid; We Are Social, S.L; 2019. Disponible en https://wearesocial.com/es/digital2019-espana. 
13. Encuesta AIMC a Usuarios de Medios de Comunicación. Madrid; Asociación para la Investigación de Medios de Comunicación, AIMC; 2018.

14. Miquel-Segarra S, Alonso-Muñoz, Marcos-García S. Buscando la interacción. Partidos y candidatos en Twitter durante las elecciones generales de 2015. Revista Prisma Social 2017;(18):34-54.

15. Quintana L, Sosa A, Castillo A. Acciones y estrategias de comunicación en plataformas digitales. El caso Cifuentes. Revista Prisma Social 2018; (22):247-270.

16. Cancelo M, Gadea G. Emponderamiento de las redes sociales en las crisis institucionales. Vivat Academia 2013;124:21-33.

17. Kearney MW. Rtweet: Collecting Twitter Data. R package version 0.6.7. Disponible en: https://cran.r-project. org/package $=$ rtweet.

18. Bastian M, Heymann S, Jacomy M. "Gephi: An Open Source Software for Exploring and Manipulating Networks". Proceedings of the Third International ICWSM Conference; 2009 17-20 mayo; San Jose: Conferencia Internacional AAAI sobre Web y Redes Sociales; 2009.
19. Brin S, Page L. The Anatomy of a Large-Scale Hypertextual Web Search Engine. Proceedings of the seventh International Conference on the World Wide Web;1998 1418 Abril; Brisbane, Australia; 1998. p.107-117.

20. Fernández Vallejo AM. (2018). Comunicar emociones en el discurso metapolítico de twitter: el caso de \#MADURO versus@NICOLASMADURO. Observatorio (OBS*) Journal 2018:175-194.

21. Sautera DA, Eisner F, Ekman P, Scott SK. Crosscultural recognition of basic emotions through nonverbal emotional vocalizations. Proc Natl Acad Sci U S A; 2010; 107(6):2408-2412.

22. Plutchik R. A general psychoevolutionary theory of emotion. Emotion: Theory, Research, and Experience 1980;1(3):3-33

23. Mohammad S, Turney P. Emotions Evoked by Common Words and Phrases: Using Mechanical Turk to Create an Emotion Lexicon. Proceedings of the NAACL-HLT 2010 Workshop on Computational Approaches to Analysis and Generation of Emotion in Text; 2010 junio; Los Angeles, California: North American Chapter of the Association for Computational Linguistics; 2010. 